\section{Uma exploração multidimensional dos componentes da síndrome metabólica}

\author{
A multidimensional exploration of metabolic \\ syndrome components
}

\author{
${ }^{1}$ Escola de Enfermagem, \\ Universidade Federal de \\ Minas Gerais, Belo Horizonte \\ Brasil. \\ 2 Escola de Medicina \\ Veterinária, Universidade \\ Federal de Minas Gerais, Belo \\ Horizonte, Brasil. \\ Correspondência \\ G. Velásquez-Meléndez \\ Departamento de \\ Enfermagem Materno- \\ Infantil e Saúde Pública, \\ Escola de Enfermagem, \\ Universidade Federal de \\ Minas Gerais. \\ Av. Alfredo Balena 190, \\ Belo Horizonte, $M G$ \\ 30130-100, Brasil. \\ guveme@ufmg.br
}

\begin{abstract}
The objective of this study was to verify the clustering of anthropometric and metabolic variables related to metabolic syndrome, by sex. Data were collected from 579 subjects aged 18-94 years living in two rural areas of Brazil. Factor analysis was performed using principal components analysis with varimax orthogonal rotation. The study reduced a complex set of cardiovascular risk factor into 3 independent factors, each reflecting a different aspect of metabolic syndrome. In both sexes, factor 1 related to obesity and dyslipidemia, factor 2 to obesity and blood pressure, and factor 3 to obesity and insulin resistance. The total variance explained for men and women was, respectively, $66.61 \%$ and $68.98 \%$. The findings corroborate the hypothesis that at least 3 pathophysiological domains act in the clustering of cardiovascular risk factors related to metabolic syndrome in this population.
\end{abstract}

Metabolic Syndrome X; Obesity; Rural Population
Eulilian Dias de Freitas 1

João Paulo Amaral Haddad 2

Gustavo Velásquez-Meléndez 1

\section{Introdução}

Desde a proposição do termo síndrome X no final dos anos 80 por Reaven 1, o agrupamento de alguns fatores de risco cardiovascular tem sido motivo de intensos debates 2 . A primeira definição proposta para a síndrome metabólica foi a da Organização Mundial da Saúde (OMS) em 19983. A partir daí, diversos organismos internacionais têm proposto alguns critérios próprios para identificar a ocorrência dessa síndrome.

Embora os critérios propostos apresentem algumas diferenças em relação à presença dos componentes e em relação ao ponto de corte dos mesmos, todos eles incluem medidas de distúrbio da homeostase da glicose, hipertensão arterial, dislipidemia e obesidade central 3,4,5,6,7. Alguns estudos têm sugerido a participação de fatores de risco não-tradicionais, por exemplo, indicadores de inflamação e indicadores prótrombóticos, como componentes da síndrome metabólica 8,9 , porém estes indicadores ainda são objeto de muitas controvérsias e não foram incluídos em nenhum dos critérios diagnósticos da síndrome metabólica.

Muitos estudos têm sido publicados a respeito da síndrome metabólica; seu reconhecimento como fator de risco para doenças cardiovasculares aumentou o interesse em se conhecer não apenas as suas causas, mas sua distribuição nas populações. Diversos estudos foram realizados em várias populações encontrando prevalências 
de $6 \%$ a 70,3\%, dependendo do grupo étnico, sexo, comorbidades associadas, critério diagnóstico utilizado, entre outros fatores 10,11,12,13.

Pesquisas mostram que a prevalência é alta também em populações rurais. A prevalência em uma população rural mexicana foi de $45,2 \%$, os autores sugerem que o motivo seja a incorporação de hábitos de vida urbanos destas populações, com maior consumo de alimentos industrializados e menor nível de atividade física 14 . No Brasil, essas prevalências variaram entre $24,8 \%$ e 19\% em dois estudos 15,16.

Há evidências de que exista mais de um processo fisiopatológico relacionado ao desenvolvimento simultâneo de alterações metabólicas, cada um produzindo um padrão de agrupamento de fatores de risco diferentes 17 . Um método estatístico utilizado para interpretar esse agrupamento de fatores de risco é a análise fatorial. Essa técnica é utilizada para agrupar um conjunto de variáveis originais em subconjuntos de novas variáveis latentes (isto é, que não podem ser medidas diretamente), chamados fatores, mutuamente não correlacionados 18. O primeiro artigo a utilizar a técnica de análise fatorial exploratória para reduzir um número maior de desordens relacionadas à síndrome metabólica a um número menor de fatores independentes foi publicado em 1994 por Edwards et al. 19. Foram obtidos três fatores que explicaram $65,8 \%$ da variância total dos dados. Desde então, muitos estudos semelhantes foram conduzidos em vários países, levando-se em conta etnia ${ }^{20}$, sexo ${ }^{21}$ e presença de co-morbidades 22 . Esses estudos têm encontrado no mínimo dois fatores 23 e a grande maioria de três a quatro fatores 20,24 .

O objetivo deste estudo foi verificar, por meio da análise fatorial, os padrões de agrupamento das variáveis antropométricas e metabólicas relacionadas com a síndrome metabólica, de acordo com o sexo, em uma população rural brasileira.

\section{Casuística e métodos}

\section{Sujeitos da pesquisa e delineamento do estudo}

Foi conduzido um estudo epidemiológico de delineamento transversal em duas comunidades rurais: Virgem da Graças, pertencente ao Município de Ponto dos Volantes; e Caju, pertencente ao Município de Jequitinhonha, ambas situadas no Vale do Jequitinhonha, Minas Gerais, Brasil. Essa região possui baixos índices de desenvolvimento humano, tendo como características marcantes um clima semi-árido e dependência da agricultura de subsistência 25 .
A população alvo deste estudo foi indivíduos com 18 anos ou mais de idade e que residiam no local há pelo menos dois anos. Foram excluídas mulheres grávidas e indivíduos que não realizaram exames bioquímicos.

\section{Coleta de dados}

Os participantes do estudo responderam a um questionário padronizado por meio de entrevista face a face. $\mathrm{O}$ instrumento incluiu perguntas relativas a aspectos sócio-econômicos, atividades diárias, estilos de vida e história obstétrica para as mulheres. Ao final da entrevista foi realizado um exame clínico que consistiu na aferição de medidas antropométricas e hemodinâmicas, além da coleta de uma amostra de sangue para dosagem de glicose, lipídeos e insulina. Com base nesses dados foram extraídas as variáveis de interesse para o presente estudo.

\section{Variáveis do estudo}

Cada medida antropométrica foi realizada três vezes por técnicos previamente treinados, segundo padrões estabelecidos por Lohman et al. 26, a média das aferições foi o valor considerado para os procedimentos de análises. A circunferência da cintura foi aferida, utilizando-se uma fita métrica inelástica, no ponto médio entre a parte inferior da costela e a parte superior da crista ilíaca, considerando-se o milímetro mais próximo. A circunferência do quadril foi aferida no local de maior protuberância da região glútea. A razão cintura-quadril (RCQ) foi obtida por meio da equação: RCQ = circunferência da cintura/circunferência do quadril e classificada como normal ( $<1$ para homens e $<0,85$ para mulheres) ou elevada ( $\geq 1$ para homens $\mathrm{e} \geq 0,85$ para mulheres), de acordo com as recomendações da OMS 27. Cabe ressaltar que no Brasil ainda não existem estudos representativos da população que estabeleçam os limites de normalidade para a RCQ, apesar de a OMS estabelecer um único ponto de corte, não levando em consideração a etnia, esse critério foi utilizado por ser amplamente aceito e para fins de comparação com outros estudos.

Para a aferição do peso corporal os sujeitos estavam vestidos com roupas leves e sem sapatos; foi utilizada uma balança digital (modelo PL 150, Filizzola Ltda., Brasil) com aproximação de $0,1 \mathrm{~kg}$. A estatura foi medida com uma fita métrica inextensível fixada em uma parede sem rodapé a uma distância de $50 \mathrm{~cm}$ do chão com aproximação de $0,1 \mathrm{~cm}$, sendo o posicionamento padronizado de pé e de costas para a parede, com os pés juntos, as nádegas, ombros e a parte posterior da cabeça tocando a fita de medição, 
os braços ao longo do corpo e a cabeça posicionada no plano de Frankfurt. O índice de massa corporal (IMC) foi calculado a partir do peso e da estatura medidos no momento da entrevista, sendo obtido por meio da seguinte equação: $\mathrm{IMC}=$ peso $(\mathrm{kg}) /$ estatura $(\mathrm{m})^{2}$.

A aferição da pressão arterial foi realizada seguindo os procedimentos preconizados pelo Joint National Committee 28, também recomendados pela IV Diretrizes Brasileiras de Hipertensão Arterial 29. O paciente permaneceu sentado durante cinco minutos, logo após este tempo a pressão arterial foi aferida usando-se o método auscultatório indireto, com esfigmomanômetro de mercúrio devidamente testado e calibrado, estando o braço apoiado na altura do precórdio. $\mathrm{O}$ primeiro som de Korotkoff foi considerado para leitura da pressão arterial sistólica e o último para leitura da pressão arterial diastólica. Três medidas foram realizadas, no braço direito, sendo a média das três a definitiva.

As amostras de sangue para exames bioquímicos foram obtidas por meio de punção venosa e distribuídas em alíquotas de acordo com as especificações técnicas para dosagem de cada uma delas com o paciente em jejum de $12 \mathrm{~h}$, sendo a coleta e a centrifugação da amostra sanguínea realizadas no local da pesquisa. O soro e o plasma de cada indivíduo foram separados em tubos de ensaios devidamente identificados e enviados ao laboratório no mesmo dia da coleta, seguindo as especificações técnicas recomendadas. No laboratório, as amostras de soro e plasma foram armazenadas em um refrigerador a $-20^{\circ} \mathrm{C}$ e analisadas usando-se um Cobas Mira Plus (Roche Diagnostics, Suíça) que estava regularmente calibrado. As concentrações de triglicérides e glicose foram determinadas utilizando-se um teste enzimático colorimétrico. A concentração da lipoproteína de alta densidade (HDL-c) também foi medida por um teste enzimático colorimétrico, após precipitação das frações (LDL-c) e (VLDL-c) pelo ácido fosfotungstico e cloreto de magnésio. A razão triglicérides/HDL-c (TG/HDL) foi utilizada como indicador de dislipidemia devido à sua relação com o incremento do risco cardiovascular 30 . As concentrações de insulina foram determinadas por um método imunométrico em fase sólida quimioluminescente usando-se o analisador Immulite 2000 (Euro/DPC Ltda., Reino Unido). $\mathrm{O}$ índice de resistência à insulina foi calculado pela fórmula do homeostasis model assessment (HOMA-IR), a partir da seguinte equação: HOMA-IR = insulinemia de jejum $(\mathrm{mU} / \mathrm{L}) \mathrm{x}$ glicemia de jejum (mmol/L)/22,5 31 .

A síndrome metabólica foi diagnosticada utilizando-se o critério estabelecido pela International Diabetes Federation em 2005 7, já que este critério é o único que estabelece pontos de corte da circunferência de cintura específicos por etnia. Apesar de os valores limítrofes ainda não serem validados por estudos brasileiros, acredita-se que os valores propostos são baseados em população semelhante à brasileira.

\section{Análise dos dados}

Os dados coletados foram analisados utilizando-se o programa Stata versão 9.0 (Stata Corp., College Station, Estados Unidos) e, para efeito de interpretação, o limite de erro tipo I foi de até $5 \%(\mathrm{p} \leq 0,05)$. As características dos participantes foram descritas segundo o sexo e a presença/ausência de síndrome metabólica, por meio de média desvio-padrão, exceto para as variáveis que foram fortemente assimétricas, quando foi utilizada a mediana (intervalo interquartílico). Para comparar as diferenças entre as médias e entre as medianas foram utilizados os testes t de Student e Mann-Whitney, respectivamente. Para verificar o grau de associação entre as variáveis, a matriz de correlação de Pearson foi utilizada.

A análise fatorial exploratória foi realizada para determinar o agrupamento dos fatores de risco cardiovasculares relacionados à síndrome metabólica. O método de extração de fatores dessa técnica é o de componentes principais e, por isto, essa técnica é também chamada de análise fatorial dos componentes principais.

A rotação varimax foi utilizada para facilitar a interpretação dos dados, mantendo uma independência dos fatores. Foram retidos na análise apenas os fatores que contribuíram com uma variância maior que a de uma variável isolada, ou seja, fatores que apresentaram autovalores maior ou igual a 1 18; para tanto foram produzidos gráficos que auxiliaram na escolha do número de fatores. Seguindo a literatura publicada nos últimos anos, cargas fatoriais (loadings) maiores ou iguais a 0,3 foram utilizadas para estabelecer a qual fator cada variável aderiu 17.

A escolha das variáveis foi baseada na matriz de correlação e nas comunalidades, sempre tendo em vista manter variáveis que contemplassem todos os componentes da síndrome metabólica: obesidade, dislipidemia, hipertensão arterial e distúrbios do metabolismo da glicose. O teste de Kaiser-Meyer-Olkin foi realizado para verificar a adequação da amostra em relação à realização da análise fatorial dos componentes principais, sendo considerado adequado se maior ou igual a 0,5.

\section{Aspectos éticos}

Este estudo foi aprovado pelo Comitê de Ética em Pesquisa em Seres Humanos da Universidade 
Federal de Minas Gerais, de acordo com a Resolução $n^{o}$. 196/96 do Conselho Nacional de Saúde. Todos os participantes foram informados a respeito dos objetivos do estudo e de seus direitos, além disto, assinaram um termo de consentimento livre e esclarecido.

\section{Resultados}

A amostra compreendeu 579 indivíduos, sendo $291(50,26 \%)$ do sexo masculino e $288(49,74)$ do feminino. As características dos participantes do estudo se encontram na Tabela 1 . A idade variou de 18 a 94 anos, não havendo diferença significativa entre os sexos. Mulheres apresentaram maiores níveis de IMC, VLDL, TG/HDL e resistência à insulina (RI) que os homens ( $\mathrm{p} \leq 0,05)$. A média da RCQ foi significativamente maior no sexo masculino. Contudo, quando essa variável é categorizada, a freqüência de RCQ acima do recomendado é significativamente maior entre as mulheres $(\mathrm{p}<0,001)$.

A prevalência total da síndrome metabólica entre os participantes do estudo, de acordo com o International Diabetes Federation (19,54\%) é significativamente maior nas mulheres que nos homens $(28,46 \%$ vs. $10,20 \%$; $\mathrm{p}<0,05)$.

As características dos participantes do estudo foram comparadas por presença/ausência de síndrome metabólica (Tabela 2). Observa-se que a mediana de idade é significativamente menor em indivíduos com síndrome metabólica. Os níveis de pressão sistólica e diastólica, bem como a RI - aferida pelo HOMA-IR - foram mais altos naqueles em que a síndrome foi diagnosticada.

A Tabela 3 apresenta a matriz de correlação dos parâmetros metabólicos medidos. Em ambos os sexos a maioria das variáveis foram significativamente correlacionadas entre si, o que viabiliza a realização da técnica de análise fatorial. Além disso, todas as associações foram positivas, tanto para o sexo masculino quanto para o feminino. Entre os homens, a pressão arterial sistólica não esteve associada à razão TG/HDL ao índice HOMA-IR e a pressão arterial diastólica também não se associou ao HOMA-IR. Já entre as mulheres apenas o componente glicemia de jejum não apresentou correlação com a razão TG/HDL e pressão arterial diastólica.

$\mathrm{Na}$ análise fatorial dos componentes principais, três componentes foram retidos, como mostra a Tabela 4. A explicação da variância total dos dados dos sexos masculino e feminino foram, respectivamente, $66,61 \%$ e $68,98 \%$. Valores de carga acima de 0,3 demonstram em que fator cada componente aderiu.

Entre os homens, o fator 1 foi caracterizado por cargas positivas de RCQ, IMC, VLDL e razão TG/HDL. No fator 2 os componentes que aderiram foram a RCQ, pressão arterial sistólica e pressão arterial diastólica, explicando $22,17 \%$ da vari-

Características gerais dos participantes do estudo.

\begin{tabular}{|c|c|c|c|c|c|c|c|}
\hline \multirow[t]{2}{*}{ Variáveis } & \multicolumn{3}{|c|}{ Masculino } & \multicolumn{3}{|c|}{ Feminino } & \multirow[t]{2}{*}{ Valor de p * } \\
\hline & $\mathrm{n}$ & $\begin{array}{l}\text { Média ou } \\
\text { mediana }\end{array}$ & $\begin{array}{c}\text { Desvio- } \\
\text { padrão ou } \\
\text { intervalo } \\
\text { interquartílico }\end{array}$ & $\mathbf{n}$ & $\begin{array}{l}\text { Média ou } \\
\text { mediana }\end{array}$ & $\begin{array}{l}\text { Desvio- } \\
\text { padrão ou } \\
\text { intervalo } \\
\text { interquartílico }\end{array}$ & \\
\hline Idade (anos) & 291 & 42,00 ** & $29,00 * \star$ & 288 & $42,00 * \star$ & $30,00 * \star$ & 0,821 \\
\hline $\mathrm{RCQ}$ & 290 & 0,89 & 0,07 & 287 & 0,85 & 0,08 & 0,000 \\
\hline $\operatorname{IMC}\left(\mathrm{kg} / \mathrm{m}^{2}\right)$ & 291 & 22,12 & 2,95 & 286 & 23,86 & 4,54 & 0,000 \\
\hline $\operatorname{VLDL}(\mathrm{mg} / \mathrm{dL})$ & 268 & 19,30 & 10,16 & 275 & 21,99 & 12,24 & 0,013 \\
\hline TG/HDL & 268 & 2,16 & 1,24 & 275 & 2,42 & 1,60 & 0,040 \\
\hline Pressão arterial sistólica (mmHg) & 291 & 136,57 & 24,11 & 288 & 139,10 & 29,58 & 0,265 \\
\hline Pressão arterial diastólica $(\mathrm{mmHg})$ & 291 & 81,89 & 13,65 & 288 & 83,99 & 15,75 & 0,091 \\
\hline HOMA-IR & 244 & 0,46 ** & $0,33 * \star$ & 252 & 0,72 ** & 1,02 ** & 0,000 \\
\hline Glicose $(\mathrm{mg} / \mathrm{dL})$ & 267 & 87,99 & 18,13 & 265 & 88,88 & 27,26 & 0,666 \\
\hline
\end{tabular}

HOMA-IR: modelo de avaliação homeostática - resistência insulina; IMC: índice de massa corporal; RCQ: razão cintura/quadril; TG/HDL: razão triglicérides/lipoproteínas de alta densidade; VLDL: lipoproteína de densidade muito baixa.

* Teste $t$ de Student/Mann-Whitney;

** Mediana e intervalo interquartílico. 
Características da amostra estudada, segundo a presença da síndrome metabólica.

\begin{tabular}{|c|c|c|c|c|c|c|c|}
\hline \multirow[t]{3}{*}{ Variáveis } & \multicolumn{6}{|c|}{ Síndrome metabólica } & \multirow[t]{3}{*}{ Valor de $p$} \\
\hline & & Ausente & & & Presente & & \\
\hline & $\mathbf{n}$ & $\begin{array}{l}\text { Média ou } \\
\text { mediana }\end{array}$ & $\begin{array}{c}\text { Desvio- } \\
\text { padrão ou } \\
\text { intervalo } \\
\text { interquartílico }\end{array}$ & n & $\begin{array}{l}\text { Média ou } \\
\text { mediana }\end{array}$ & $\begin{array}{c}\text { Desvio- } \\
\text { padrão ou } \\
\text { intervalo } \\
\text { interquartílico }\end{array}$ & \\
\hline Idade (anos) * & 411 & 43,00 & 29,00 & 99 & 38,00 & 33,00 & 0,000 \\
\hline $\mathrm{RCQ}$ & 411 & 0,87 & 0,08 & 98 & 0,87 & 0,08 & 0,593 \\
\hline $\mathrm{IMC}\left(\mathrm{kg} / \mathrm{m}^{2}\right)$ & 410 & 22,94 & 3,94 & 98 & 23,09 & 3,70 & 0,738 \\
\hline VLDL (mg/dL) & 383 & 20,74 & 10,64 & 95 & 19,85 & 11,88 & 0,486 \\
\hline $\mathrm{TG} / \mathrm{HDL}$ & 383 & 2,25 & 1,29 & 95 & 22,64 & 1,65 & 0,938 \\
\hline Pressão arterial sistólica $(\mathrm{mmHg})$ & 411 & 137,37 & 26,65 & 99 & 140,50 & 29,58 & 0,307 \\
\hline Pressão arterial diastólica (mmHg) & 411 & 82,60 & 14,76 & 99 & 84,02 & 15,59 & 0,403 \\
\hline HOMA-IR * & 350 & 0,51 & 0,72 & 89 & 0,55 & 0,65 & 0,024 \\
\hline Glicose (mg/dL) & 373 & 89,01 & 26,24 & 94 & 87,26 & 14,85 & 0,531 \\
\hline
\end{tabular}

HOMA-IR: modelo de avaliação homeostática - resistência insulina; IMC: índice de massa corporal; RCQ: razão cintura-quadril; TG/HDL: razão triglicérides/lipoproteínas de alta densidade; VLDL: lipoproteína de densidade muito baixa.

* Mediana/intervalo interquartílico.

Tabela 3

Matriz de correlação dos componentes da síndrome metabólica, por sexo.

\begin{tabular}{|c|c|c|c|c|c|c|c|c|}
\hline Masculino/ Feminino & $\mathrm{RCO}$ & IMC & VLDL & TG/HDL & $\begin{array}{c}\text { Pressão } \\
\text { arterial } \\
\text { sistólica }\end{array}$ & $\begin{array}{c}\text { Pressão } \\
\text { arterial } \\
\text { diastólica }\end{array}$ & HOMA-IR & Glicemia \\
\hline $\mathrm{RCQ}$ & - & 0,49 * & 0,40 * & 0,38 * & 0,39 * & 0,29 * & 0,20 * & 0,22 * \\
\hline $\mathrm{IMC}\left(\mathrm{kg} / \mathrm{m}^{2}\right)$ & 0,44 * & - & 0,22 * & 0,23 * & 0,18 * & 0,15 * & 0,20 * & 0,23 * \\
\hline $\operatorname{VLDL}(\mathrm{mg} / \mathrm{dL})$ & 0,34 * & 0,33 * & - & 0,92 * & 0,22 * & 0,15 * & 0,20 * & $0,13 * \star$ \\
\hline TG/HDL & 0,30 * & 0,35 * & 0,80 * & - & 0,18 * & 0,13 * & 0,15 * & 0,10 \\
\hline Pressão arterial sistólica $(\mathrm{mmHg})$ & 0,21 * & $0,10 * *$ & 0,14 ** & 0,09 & - & 0,76 * & 0,20 * & 0,16 * \\
\hline Pressão arterial diastólica $(\mathrm{mmHg})$ & 0,23 * & 0,18 * & 0,19 * & $0,14 * \star$ & 0,67 * & - & $0,13 * \star$ & 0,08 \\
\hline HOMA-IR & 0,21 * & 0,28 * & 0,25 * & 0,26 * & 0,03 & 0,04 & - & 0,16 * \\
\hline Glicemia (mg/dL) & 0,15 * & 0,14 ** & 0,17 * & 0,17 * & 0,19 * & 0,14 ** & 0,25 * & - \\
\hline
\end{tabular}

HOMA-IR: modelo de avaliação homeostática - resistência insulina; IMC: índice de massa corporal; RCQ: razão cintura-quadril; TG/HDL: razão triglicérides/lipoproteínas de alta densidade; VLDL: lipoproteína de densidade muito baixa.

* $p<0,01$;

$\star * * 0,05$.

ância dos dados. Já o fator 3 foi composto por cargas positivas de RCQ, IMC, glicemia e HOMA-IR; explicou 17,98\% da variância dos dados.

Os resultados encontrados para as mulheres foram semelhantes aos dos homens. No fator 1 os componentes RCQ, VLDL e TG/HDL aderiram positivamente; explicando $25,62 \%$ da variância dos dados. O fator 2 foi carregado positivamen- te por RCQ, pressão arterial sistólica e pressão arterial diastólica; a explicação da variância dos dados deste fator foi de $23,32 \%$. Por fim, o fator 3 foi caracterizado por RCQ, IMC, glicemia de jejum e HOMA-IR; este fator explicou $20,04 \%$ da variância dos dados.

A RCQ esteve associada positivamente a todos os três fatores retidos pela análise fatorial, 
Análise fatorial dos componentes principais, após rotação varimax dos componentes da síndrome metabólica, por sexo.

\begin{tabular}{|c|c|c|c|c|c|c|}
\hline \multirow[t]{2}{*}{ Variável } & \multicolumn{3}{|c|}{ Masculino } & \multicolumn{3}{|c|}{ Feminino } \\
\hline & Fator 1 & Fator 2 & Fator 3 & Fator 1 & Fator 2 & Fator 3 \\
\hline $\mathrm{RCQ}$ & 0,44 & 0,31 & 0,38 & 0,35 & 0,33 & 0,58 \\
\hline IMC (kg/m²) & 0,49 & 0,09 & 0,46 & 0,15 & 0,08 & 0,75 \\
\hline $\operatorname{VLDL}(\mathrm{mg} / \mathrm{dL})$ & 0,90 & 0,10 & 0,07 & 0,96 & 0,10 & 0,09 \\
\hline TG/HDL & 0,90 & 0,03 & 0,09 & 0,97 & 0,07 & 0,07 \\
\hline Pressão arterial sistólica (mmHg) & 0,03 & 0,90 & 0,05 & 0,13 & 0,92 & 0,13 \\
\hline Pressão arterial diastólica $(\mathrm{mmHg})$ & 0,12 & 0,89 & 0,02 & 0,05 & 0,94 & 0,02 \\
\hline Glicemia (mg/dL) & $-0,02$ & 0,20 & 0,71 & $-0,01$ & 0,03 & 0,66 \\
\hline HOMA-IR & 0,20 & $-0,09$ & 0,75 & 0,13 & 0,14 & 0,48 \\
\hline Variância total (\%) & 26,46 & 22,17 & 17,98 & 25,62 & 23,32 & 20,04 \\
\hline Variância acumulada (\%) & 26,46 & 48,63 & 66,61 & 25,62 & 48,94 & 68,98 \\
\hline
\end{tabular}

HOMA-IR: modelo de avaliação homeostática - resistência insulina; IMC: índice de massa corporal; RCQ: razão cintura-quadril; TG/HDL: razão triglicérides/lipoproteínas de alta densidade; VLDL: lipoproteína de densidade muito baixa.

Nota: destacados em negrito os valores de carga acima de 0,3 que demonstram em que fator cada componente aderiu.

tanto em homens quanto em mulheres. Já o IMC aderiu aos fatores 1 e 3 no modelo encontrado para os homens e apenas ao fator 3 no modelo das mulheres (Figura 1).

A adequação da amostra para o modelo de análise fatorial foi satisfatória, tanto para o sexo masculino (Kaiser-Meyer-Olkin $=0,66$ ) quanto para o feminino (Kaiser-Meyer-Olkin $=0,62$ ).

\section{Discussão}

No presente estudo a análise fatorial foi utilizada para caracterizar o agrupamento dos componentes da síndrome metabólica em homens e mulheres maiores de 18 anos residentes em áreas rurais. Com essa técnica, oito fenótipos, componentes da síndrome metabólica da matriz de dados estudada, foram reduzidos a três fatores independentes, tanto para homens quanto para mulheres, que explicaram $66,61 \%$ e $68,98 \%$ da variância total destes dados, respectivamente. Observou-se três dimensões distintas e independentes que seriam a base do agrupamento desses fatores de risco cardiovasculares. Os fatores retidos apresentaram discretamente um componente em comum: a obesidade central, medida pela RCQ.

Recentemente, a American Heart Association e o National Heart, Lung, and Blood Institute passaram a reconhecer três potenciais bases etiológicas para a síndrome metabólica ${ }^{32}$. Além da RI, a obesidade e distúrbios do tecido adiposo, uma variedade de fatores independentes que incluem moléculas de origem hepática - como a proteína
C-reativa-de origem vascular e imunológica passaram a ser considerados importantes na patogênese da síndrome metabólica. Reconheceram também a existência de uma grande variedade de fatores contribuintes, tais como idade, estado pró-inflamatório e mudanças hormonais.

Os padrões de agrupamento neste estudo foram semelhantes entre homens e mulheres, exceto pelo IMC que no sexo masculino esteve aderido ao fator 1 e ao fator 3 , e nas mulheres se aderiu apenas ao fator 3 .

O primeiro fator foi nomeado dislipidemia/ obesidade e explicou $26,46 \%$ e $25,62 \%$ da variância da matriz dos dados nos modelos masculino e feminino, respectivamente. Esse fator é carregado fortemente pelas variáveis VLDL e TG/HDL, e em menor medida pelo IMC e RCQ nos homens e apenas pelo RCQ, nas mulheres; o que indica que a dislipidemia é o componente mais fortemente aderido a esse fator, e considerado o fator principal da matriz de dados. Os valores das cargas das medidas de obesidade foram maiores nos homens, o que indica que a associação entre este componente e dislipidemia foi mais forte no sexo masculino que no feminino.

Semelhante aos achados deste estudo, Snehalatha et al. 17, estudando a população indiana encontraram diferenças no fator carregado pelo componente dislipidemia entre os sexos. Nos homens, as medidas de colesterol total e triglicérides aderiram ao mesmo fator que o IMC e RCQ, enquanto no modelo extraído para as mulheres a dislipidemia formou um domínio independente das outras variáveis. 


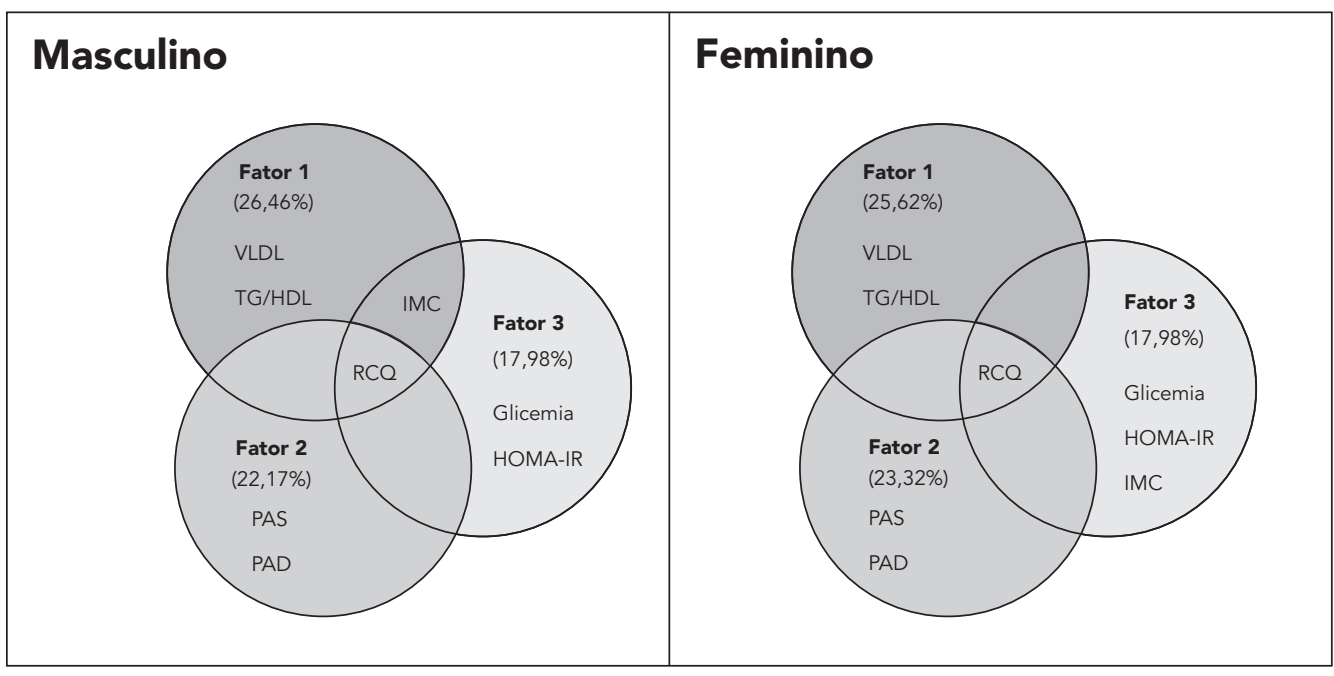

HOMA-IR: modelo de avaliação homeostática - resistência insulina; IMC: índice de massa corporal; RCQ: razão cinturaquadril; TG/HDL: razão triglicérides/lipoproteínas de alta densidade; VLDL: lipoproteína de densidade muito baixa. Nota: destacados em negrito os valores de carga acima de 0,3 que demonstram em que fator cada componente aderiu.

A maioria dos trabalhos que realizaram análise fatorial encontrou pressão arterial sistólica e pressão arterial diastólica como um fator isolado 24 . Neste estudo, o segundo fator foi composto por níveis da pressão arterial e obesidade abdominal - sendo nomeado hipertensão/obesidade - porém, as cargas da RCQ, tanto no modelo masculino quanto no feminino, foram muito próximas de 0,3. Esses resultados corroboram com os obtidos por Ang et al. 33 , que também encontraram os níveis de pressão arterial e medidas de obesidade aderidos ao mesmo fator.

Um estudo realizado na população chinesa sugere que os níveis pressóricos não sejam um componente da síndrome metabólica. Esses autores realizaram a análise fatorial incluindo e excluindo medida de pressão arterial, no primeiro caso, isolaram dois fatores independentes e, no segundo, apenas um fator; para eles, esses resultados sugerem que a síndrome metabólica possui um fator que seja sua base 34 .

Por fim o terceiro fator, nomeado distúrbios de homeostase da glicose/obesidade, foi carregado por medidas de HOMA-IR, glicemia de jejum e de obesidade, sendo que o IMC apresentou uma carga maior do que a RCQ, em ambos os sexos. A RI, hiperglicemia e medidas de obesidade (principalmente obesidade geral, medida pelo IMC) também estiveram correlacionadas em um mesmo fator na população masculina e feminina do Sul da Índia 17.

Ao contrário do que foi sugerido por outros autores em outras populações, a RI não foi o componente principal do agrupamento da matriz de dados estudada, pois, no modelo final, três fatores independentes foram retidos. Autores que consideram a RI como fator mais importante que a obesidade na patogênese da síndrome metabólica argumentam que a RI, ou a hiperinsulinemia, seja a causa direta dos outros fatores de risco metabólico 35 . No entanto, deve-se ter cautela ao identificar a RI como causa principal da síndrome metabólica, já que a primeira está intrinsecamente associada à obesidade.

Existe abundante controvérsia na definição da síndrome metabólica e sobre quais seriam os componentes mais adequados a serem incorporados na definição, isto certamente é decorrente da grande possibilidade de combinações dos mesmos. Estudos de predição para eventos cardiovasculares indicam que a hiperglicemia, níveis baixos de HDL e hipertensão arterial conferem riscos elevados de eventos cardiovasculares maiores que a obesidade e hipertrigliceridemia 36,37 . Em outro estudo, quando o evento cardiovascular foi o grau de aterosclerose da carótida, os 
triglicérides e a pressão arterial foram melhores preditores ${ }^{38}$. Além disso, há evidencias de que as alterações do metabolismo de carboidratos, HDL e microalbuminuria conferem maior grau de predição para morte por doença cardiovascular 39 .

A análise fatorial exploratória tem sido alvo de críticas, alguns autores têm afirmado categoricamente a necessidade de se utilizar a análise fatorial confirmatória, já que esta testa hipótese e possui critérios mais rígidos de análise 40 . Contudo, a importância da análise fatorial exploratória, utilizada neste estudo, reside na capacidade de gerar hipóteses 41 , que poderão ser confirmadas a posteriori, principalmente em populações nas quais não se tem resultados consolidados, populações que ainda não foram estudas e quando não se conhece a estrutura de agrupamento dos fatores - como é o caso da população rural brasileira.

Para verificar a consistência dos achados deste estudo foram realizadas análises semelhantes a esta em duas subamostras aleatórias (50\%), tendo sido encontrados resultados semelhantes.

\section{Resumo}

O objetivo deste estudo foi verificar os padrões de agrupamento das variáveis antropométricas e metabólicas relacionadas com a síndrome metabólica, de acordo com o sexo. Foram coletados dados de 579 moradores de duas comunidades rurais brasileiras, com idades entre 18 e 94 anos. A análise fatorial foi realizada utilizando-se o método de extração de componentes principais e rotação ortogonal varimax. Este estudo reduziu um grupo complexo de fatores de risco cardiovascular que caracterizam a síndrome metabólica em três fatores independentes, cada um refletindo um aspecto diferente da síndrome metabólica. Em ambos os sexos, o fator 1 esteve relacionado à obesidade e dislipidemia, o fator 2 à obesidade e pressão arterial e o fator 3 à obesidade e resistência à insulina. As variâncias totais explicadas para os sexos masculino e feminino foram, respectivamente, 66,61\% e 68,98\%. Esses achados corroboram com a hipótese de que pelo menos três processos fisiopatológicos atuam no agrupamento dos fatores de risco cardiovasculares na população rural estudada.

Síndrome X Metabólica; Obesidade; População Rural
No Brasil, não foram encontrados estudos que utilizaram esta técnica, seja em população rural ou urbana. Embora haja diferenças na composição da amostra quanto à raça, sexo, idade, número de variáveis incluídas, tamanhos da amostra e o ponto de corte utilizado em outras populações, os resultados deste trabalho são semelhantes a estudos realizados em diferentes partes do mundo.

A amostra estudada pode não ser considerada representativa da população brasileira devido a suas características específicas. Contudo, acredita-se que estes resultados refletem o agrupamento dos fatores de risco cardiovasculares considerados componentes da síndrome metabólica.

Concluindo, os resultados apresentados corroboram com a hipótese de que pelo menos três processos fisiopatológicos atuam no agrupamento dos fatores de risco cardiovasculares na amostra rural estudada, confirmando em populações rurais do Brasil, resultados obtidos em populações urbanas e rurais de outros países.

\section{Colaboradores}

E. D. Freitas colaborou na revisão bibliográfica, análises estatísticas e redação do artigo. J. P. A. Haddad contribuiu nas análises estatísticas e redação do manuscrito. G. Velásquez-Meléndez colaborou no planejamento, análise e redação do artigo.

\section{Agradecimentos}

Ao apoio financeiro da Fundação de Amparo à Pesquisa do Estado de Minas Gerais (FAPEMIG) e do Conselho Nacional de Desenvolvimento Científico e Tecnológico (CNPq). A Dra. Andrea Gazzinelli pela sua colaboração na organização e gestão da coleta de dados. 


\section{Referências}

1. Reaven GM. Banting lecture 1988. Role of insulin resistance in human disease. Diabetes 1988; 37:1595-607.

2. Kahn R, Buse J, Ferrannini E, Stern M. The metabolic syndrome: time for a critical appraisal. Diabetologia 2005; 48:1684-99.

3. Alberti KG, Zimmet PZ. Definition, diagnosis and classification of diabetes mellitus and its complications. Part 1: diagnosis and classification of diabetes mellitus provisional report of a WHO consultation. Diabet Med 1998; 15:539-53.

4. Expert Panel on Detection, Evaluation, and Treatment of High Blood Cholesterol in Adults. Executive Summary of the Third Report of the National Cholesterol Education Program (NCEP) Expert Panel on Detection, Evaluation, and Treatment of High Blood Cholesterol in Adults (Adult Treatment Panel III). JAMA 2001; 285:2486-97.

5. Balkau B, Charles MA, Drivsholm T, Borch-Johnsen K, Wareham N, Yudkin JS, et al. Frequency of the WHO metabolic syndrome in European cohorts, and an alternative definition of an insulin resistance syndrome. Diabetes Metab 2002; 28:364-76.

6. Einhorn D, Reaven GM, Cobin RH, Ford E, Ganda OP, Handelsman Y, et al. American College of Endocrinology position statement on the insulin resistance syndrome. Endocr Pract 2003; 9:237-52.

7. International Diabetes Federation. The IDF consensus worldwide definition of the Metabolic Syndrome. http://www.idf.org/webdata/docs/ IDF_Metasyndrome_definition.pdf (acessado em 15/Set/2006).

8. Heliövaara MK, Teppo AM, Karonen SL, Tuominen JA, Ebeling P. Plasma IL-6 concentration is inversely related to insulin sensitivity, and acute-phase proteins associate with glucose and lipid metabolism in health subjects. Diabetes Obes Metab 2005; 7:729-36.

9. Ford ES. The metabolic syndrome and C-reactive protein, fibrinogen, and leukocyte count: findings from the Third National Health and Nutrition Examination Survey. Atherosclerosis 2003; 168:351-8.

10. Meigs JB, Wilson PW, Nathan DM, D'Agostinho RB, Williams K, Halner SM. Prevalence and characteristics of the metabolic syndrome in the San Antonio Heart Study and Framingham Offspring Studies. Diabetes 2003; 52:2160-7.

11. Chapidze G, Dolidze N, Enquobahrie DA, Kapanadze S, Latsabidze N, William MA. Metabolic syndrome and C-reactive protein among cardiology patients. Arch Med Res 2007; 38:783-8.

12. Moebus S, Hanisch JU, Aidelsburger P, Bramlage P, Wasem J, Jöeckel KH. Impact of 4 different definitions used for the assessment of the prevalence of the metabolic syndrome in primary healthcare: The German Metabolic and Cardiovascular Risk Project. Cardiovasc Diabetol 2007, 6:22.

13. Ford ES, Giles WH, Dietz WH. Prevalence of the metabolic syndrome among US adults: findings from the third national health and nutrition examination survey. JAMA 2002; 287:356-9.
14. Pinto ME, Lomelí AH, Gambá MAA, Flores HM, Mellado AV. Síndrome metabólico en adultos de 20 a 40 años en una comunidad rural mexicana. Rev Méd Inst Mex Seguro Soc 2006; 44:329-35.

15. Oliveira EP, Souza MLA, Lima MDA. Prevalência de síndrome metabólica em uma área rural do semiárido baiano. Arq Bras Endocrinol Metabol 2006; 50:456-65.

16. Velásquez-Meléndez G, Gazzinelli A, CôrreaOliveira R, Pimenta AM, Kac G. Prevalence of metabolic syndrome in a rural area of Brazil. São Paulo Med J 2007; 125:155-62.

17. Snehalatha C, Sivasankari S, Satyavani K, Vijay V, Ramachandran A. Insulin resistance alone does not explain the clustering of cardiovascular risk factors in southern India. Diabet Med 2000; 17:152-7.

18. Mingoti AS. Análise de dados através de métodos de estatística multivariada: uma abordagem aplicada. Belo Horizonte: Editora da Universidade Federal de Minas Gerais; 2005.

19. Edwards KL, Austin MA, Newman B, Mayer E, Krauss RM, Selby JV. Multivariate analysis of the insulin resistance syndrome in women. Arterioscler Thromb1994; 14:1940-5.

20. Shen BJ, Goldberg RB, Llabre MM, Schneiderman N. Is the factor structure of the metabolic syndrome comparable between men and women and the across three ethnic groups: the Miami Community Health Study. Ann Epidemiol 2006; 16:131-7.

21. Lafortuna CL, Adorni F, Agosti F, Sartorio A. Factor analysis of metabolic syndrome components in obese women. Nutr Metab Cardiovasc Dis 2008; 18:233-41.

22. Shmulewitz D, Auerbach SB, Lehner T, Blundell ML, Winick JD, Youngman LD, et al. Epidemiology and factor analysis of obesity, type II diabetes, hypertension, and dyslipidemia (syndrome $\mathrm{X}$ ) on the island of Kosrae, Federated States of Micronesia. Hum Hered 2001; 51:8-19.

23. Lin JD, Wan HL, Li JC, Wu CZ, Kuo SW, Hsieh CH, et al. Impaired glucose tolerance and impaired fasting glucose share similar underlying pathophysiologies. Tohoku J Exp Med 2007; 212:349-57.

24. Maison P, Day NE, Byrne CD, Wareham NJ, Hales CN. Do different dimensions of the metabolic syndrome change together over time? Diabetes Care 2001; 24:1758-63.

25. Kloss H, Passos LK, Lo-Verde P, Oliveira AC, Gazzinelli A. Distribution and Schistosoma mansoni infection of Biomphalaria glabrata in different habitants in a rural area in the Jequitinhonha Valley, Minas Gerais, Brazil: environmental and epidemiological aspects. Mem Inst Oswaldo Cruz 2004; 99:673-80

26. Lohman TG, Roche AF, Martorell R. Anthropometric standardization reference manual. Champaign: Human Kinetics; 1988.

27. World Health Organization. Obesity: preventing and managing the global epidemic. Geneva: World Health Organization; 1998. (WHO Technical Report Series, 894). 
28. The sixth report of the Joint National Committee on prevention, detection, evaluation, and treatment of high blood pressure. Arch Intern Med 1997; 157:2413-46.

29. Sociedade Brasileira de Hipertensão. IV diretrizes brasileiras de hipertensão arterial. http://www. sbh.org.br/documentos/index.asp (acessado em 09/Out/2006)

30. McLaughlin T, Abbasi F, Cheal K, Chu J, Lamendola C, Reaven G. Use of metabolic markers to identify overweight individuals who are insulin resistant. Ann Intern Med 2003; 139:802-9.

31. Matthews DR, Hosker JP, Rudenski AS, Naylor BA, Treacher DF, Turner RC. Homeostasis model assessment: insulin resistance and beta-cell function from plasma glucose and insulin concentrations in man. Diabetologia 1985; 28:412-9.

32. Grundy SM, Brewer HB, Cleeman JI, Smith SC, Lenfant C. Definition of metabolic syndrome: report of the National Heart, Lung, and Blood Institute/American Heart Association conference on scientific issues related to definition. Circulation 2004; 109:433-8.

33. Ang LW, Ma S, Cutter J, Chew SK, Tan CE, Tai ES. The metabolic syndrome in Chine, Malays and Asians Indians: factor analysis of data from the 1998 Singapore National health survey. Diabetes Res Clin Pract 2005; 67:53-62.

34. Anderson PJ, Critchley JAJH, Chan JCN, Cockram CS, Lee ZSK, Thomas GN, et al. Factor analysis of the metabolic syndrome: obesity vs. insulin resistance as the central abnormality. Int J Obes 2001; 25:1782-8.
35. Ferrannini E, Haffner SM, Mitchell BD, Stern MP Hyperinsulinaemia: the key feature of a cardiovascular and metabolic syndrome. Diabetologia 1991; 34:416-22.

36. Alexander CM, Landsman PB, Teutsch SM, Haffner SM; Third National Health and Nutrition Examination Survey (NHANES III); National Cholesterol Education Program (NCEP). NCEP-defined metabolic syndrome, diabetes, and prevalence of coronary heart disease among NHANES III participants age 50 years and older. Diabetes 2003; 52:1210-4.

37. Hunt KJ, Resendez RG, Williams K, Haffner SM, Stern MP. National Cholesterol Education Program versus World Health Organization metabolic syndrome in relation to all-cause and cardiovascular mortality in the San Antonio Heart Study. Circulation 2004; 110:1251-7.

38. Golden SH, Folsom AR, Coresh J, Sharrett AR, Szklo M, Brancati F. Risk factor groupings related to insulin resistance and their synergistic effects on subclinical atherosclerosis: the atherosclerosis risk in communities study. Diabetes 2002; 51:3069-76.

39. Wang J, Ruotsalainen S, Moilanen L, Lepistö P, Laakso M, Kuusisto J. The metabolic syndrome predicts cardiovascular mortality: a 13-year followup study in elderly non-diabetic Finns. Eur Heart J 2007; 28:857-64.

40. Pladevall M, Singal B, Williams LK, Brotons C, Guyer $\mathrm{H}$, Sadurni J, et al. A single factor underlies the metabolic syndrome. Diabetes Care 2006; 29:113-22.

41. Tang W, Pankow JS, Arnett DK. Re: (Mis) use of factor analysis in the study of insulin resistance syndrome. Am J Epidemiol 2005; 162:921-2.

Recebido em 15/Mai/2008

Versão final reapresentada em 21/Jan/2009

Aprovado em 22/Jan/2009 